\title{
A more social science: barriers and incentives for scientists engaging in policy
}

\author{
Gerald G Singh $^{1 * \dagger}$, Jordan Tam ${ }^{1 \dagger}$, Thomas D Sisk ${ }^{2}$, Sarah C Klain ${ }^{1}$, Megan E Mach ${ }^{1}$, Rebecca G Martone ${ }^{1}$, \\ and Kai MA Chan ${ }^{1}$
}

Scientists are increasingly called upon to engage in policy formulation, but the literature on engagement is strong on speculation and weak on evidence. Using a survey administered at several broadly "ecological" conferences, we investigated: (1) the extent to which respondents engage in policy-related activities (including reporting scientific results, interpreting science for policy makers, integrating science into decision making, taking a position on a policy issue, and acting as a decision maker); (2) what factors best explain these types of engagement; and (3) whether respondents' activity levels match their stated beliefs on such activities. Different factors explain different forms of participation. Past negative experience was identified as a barrier to taking part in policy, while self-perceived competence in navigating the science-policy interface was consistently important in explaining activity across all engagement types, highlighting the importance of training programs linking scientists to policy. Many respondents believed that scientists should interpret, integrate, and advocate, which contrasts with previous research and relatively low levels of self-reported participation in policy.

Front Ecol Environ 2014; 12(3): 161-166, doi:10.1890/130011 (published online 7 Feb 2014)

B oth scientists and policy makers are increasingly recognizing the need to incorporate the best available science into public policy making. The Ecological Society of America, the Society for Conservation Biology, and many other scientific societies identify public education and policy engagement as central to their missions. Science is often invoked by policy makers to validate their agendas, and scientists engage in policyrelated activities through a variety of avenues (Panel 1; Scott et al. 2007).

Given the centrality of the science-policy interface in many pressing global issues (eg climate change, desertification, biodiversity loss), it is not surprising that scientists' "engagement" is the focus of continuing debate. On one side of this often polarized discussion, some have called for scientists to avoid direct engagement by appealing to ideals of objectivity, the integrity of science, and disinterested inquiry (Nielsen 2001), while others have described the need to clearly delineate what role the scientist is playing (Lackey 2007): for instance whether an individual is acting in the capacity of a scientist or as a citizen. At the other extreme, many have insisted that scientists openly advocate for pro-environmental positions (Noss 2007).

Amid this debate, there has been a proliferation of training opportunities, awards, and fellowships to facilitate scientists' engagement with the public and with policy makers (eg the Leopold Leadership Program, COMPASS). In addition, many people have asked for amended

${ }^{1}$ Institute for Resources, Environment and Sustainability, University of British Columbia, Vancouver, Canada*'(gsingh@ires.ubc.ca); ${ }^{2}$ Landscape Conservation Initiative, School of Earth Sciences and Environmental Sustainability, Northern Arizona University, Flagstaff, AZ; 'these authors contributed equally to this paper incentive structures to encourage engagement, such as evaluating a scientist's contribution using metrics that capture impacts beyond academia and including clearly stated expectations of public outreach and policy engagement in tenure and promotion reviews (Chan et al. 2005). However, promoting such programs and incentives has been largely based on assumptions about what encourages and impedes scientists' engagement, with little empirical evidence (some exceptions are described below).

Several influential factors have been empirically tested in the literature and are briefly summarized here. Sociodemographic variables (eg age, academic seniority) contribute to engagement activity (Royal Society 2006; von Roten 2011). Personal beliefs and underlying assumptions about the nature of science - such as whether science is the only way of achieving valid knowledge - also correlate with levels of engagement (Poliakoff and Webb 2007). Former adverse experiences with policy-related activities are negatively associated with current engagement (Poliakoff and Webb 2007). Other variables may influence scientists' participation in policy but have not been verified empirically. Confidence and training are thought to be positively associated with involvement in policy, as is time availability (Royal Society 2006), holding tenure (Whitmer et al. 2010), and having institutional and peer support (Poliakoff and Webb 2007). Given the demonstrated and hypothesized role of these variables as expressed in the literature, we investigated the importance of each in explaining actual levels of policy engagement.

To move the debate away from controversial assertions about whether scientists "should or should not engage", we recognize that scientists face complex choices in a context of often-contradictory incentives and con- 
straints. Furthermore, these choices pertain to engagement activities that differ greatly in nature (Pielke 2007) and cannot be properly understood in aggregate. Here, we empirically investigated the following related questions: (1) to what extent do scientists engage, (2) how does this compare with their expressed beliefs about how scientists should engage, and (3) what factors are most closely associated with different types of policy engagement?

\section{Methods}

Survey questionnaires were distributed at nine conservation- and ecology-related conferences - held by organizations with regional to global membership - that focused on ecology, environment, and land or natural resource management $(n=508$ respondents; see WebTables 1 and 2 for respondents' country of residence and for conferences, respectively). Respondents were conference attendees, with a preponderance of ecologists and environmental scientists

\section{Panel 1. Five selected avenues in which scientists engage in policy}

- Reporting only: reporting science, which includes publishing their findings, presenting their work at conferences, etc.

- Interpreting: interpreting science for policy makers and the public without taking a policy position (eg publishing an article in the popular media, being interviewed by a journalist, giving a public seminar).

Integrating: integrating science into decision making through collaborations with policy makers without taking a policy position (eg providing expert advice, writing science briefs or white papers).

- Taking a position: actively supporting a position on a particular issue based on scientific results (eg participating in an organized campaign, writing op-eds, contacting politicians to voice opinions, speaking at rallies or protests).

- Decision making: acting as a decision maker with regard to policy.

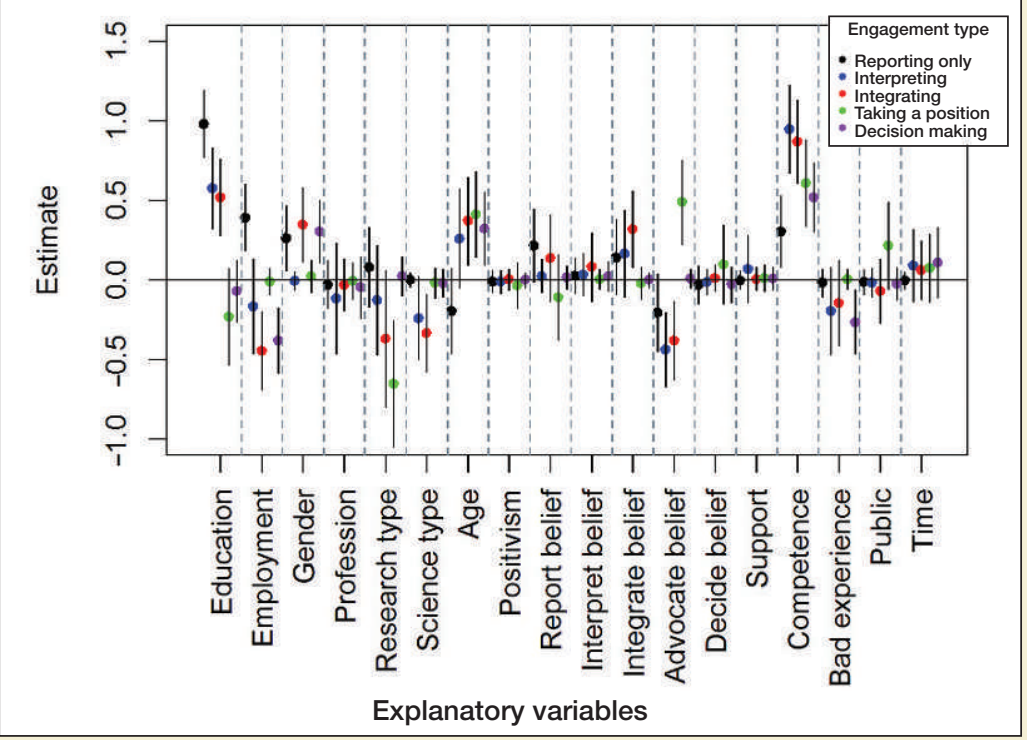

Figure 1. The 18 variables explaining participant engagement in policy, across the five engagement response variables, appear on the $x$ axis. The modelaveraged standardized regression coefficients and their $95 \%$ confidence intervals, based on the top 5000 models from all 262144 candidate models, are displayed. Significant variables are those whose confidence intervals do not cross zero.
(WebFigure 1). Our study was broader and more compreompare how multiple factors contribute to engagement.

We addressed involvement in policy following the categories developed by Steel et al. (2004), by asking particiants to indicate the frequency with which they underfive selected actions to link science with policy: decision making (see Panel 1 and Figure 1). WebTable 3. We used five-point Likert scales for all variables other than those dealing with sociodemographics. We concluded the survey with questions about conditions under which respondents would or would not promote a policy direction (eg would they support a policy ientific support that was contrary to their values?).

Correlation analyses and scatterplots were used to assess potential collinearity among explanatory variables (we found none). We used correlation, principal components analysis, and non-metric multidimensional scaling to reduce the number of variables (see WebTable 4 for index construction). The "positivism" variable - indicating the degree to which a respondent thinks valid knowledge can only be acquired through science - was also an index adapted from the literature (Gray and Campbell 2009). To aid in interpreting the resulting regression coefficients (see analysis description below), we put all explanatory variables on a common scale by centering (subtracting the mean) and dividing each by two standard deviations (Gelman 2008). For ease in computing, we transformed some of the categorical sociodemographic variables to dichotomous variables.

To quantify the relative effects of each of the 18 explanatory variables (WebTable 3 ) on each of our response variables (the five types of engagement), we used an information-theoretic approach (comparing models based on how well they account for information in the data; Burnham and Anderson 2002) to assess the 262144 candidate models that comprised all possible combinations of the explanatory variables. This approach examines the exhaustive set of models that can be constructed given the independent variables identified, and considers the fit of each model to the data. For each model, we used ordinary least squares multiple regression and calculated 
Akaike weights based on the small sample size corrected Akaike's Information Criterion (AICc), a metric that balances model fit and complexity (Burnham and Anderson 2002); these weights were used to calculate importance values and model-averaged coefficients for each variable. Owing to the large number of models and computing limitations, we tried model averaging across the best 100 , 1000,2000 , and 5000 models. We found that results were consistent (even between 1000 and 5000 models; WebFigure 2), and all results presented here are based on model averaging across the best (highest AICc weights) 5000 models (WebTable 5).

We conducted additional analyses on the subsample of respondents who were professors $(n=60)$ to see how tenure status affected their policy engagement. We removed from the model those variables that were consistent among tenured and non-tenured professors (education level, place of employment, and position) and then included tenure as a dichotomous variable. Including all 16 variables, we again conducted a model averaging procedure across the best 5000 models for each of our measures of engagement using AICc to see whether, and how, tenure was related to types of engagement. For all analyses we used the glmulti package in R (Calcagno 2012).

\section{Results and discussion}

The variables included in this study have been identified as, or hypothesized to be, important elsewhere (Steel et al. 2004; Royal Society 2006; Poliakoff and Webb 2007), but previous studies have generally considered a smaller number of independent variables and single "lumped" measures of engagement. Our set of predictor variables is not comprehensive and our study is limited by the selfreported nature of the responses; nevertheless, the information-theoretic approach allows an understanding of the relative importance of factors across engagement types. This is the first instance, to our knowledge, of an analysis of the relative importance of variables contributing to engagement. Not surprisingly, we found that different engagement activities are influenced by different factors; however, our analysis allows a more thorough exploration of these nuances than was possible in previous studies.

\section{Different drivers influence different engagement activities}

Our results reinforce the important notion that there is a spectrum of policy-engagement activities (Lach et al. 2003) by showing that our independent variables influence the (self-reported) frequency of participation in different types of engagement in different ways (Panel 1 and Figure 1). Below we report the strongest predictor variables (the 95\% confidence intervals of which do not cross zero) for each type of engagement.

The strongest predictors for reporting science were having a $\mathrm{PhD}$, working at a university, possessing a high level of self-perceived competence, and being male. For interpreting science, self-perceived competence and holding a $\mathrm{PhD}$ were strong positive predictors, while believing that scientists should advocate had a strong negative relationship; this suggests that respondents who believe scientists should take a position interpret science less frequently. For integrating science, having self-perceived competence, holding a $\mathrm{PhD}$, being male, being older, and believing that scientists should integrate all had strong positive associations, while working at a university or college, conducting "pure, basic research", and believing that scientists should advocate had negative associations. For actively taking a position (ie advocating), high self-perceived competence, a strong belief that scientists should advocate, and being older had strong positive associations, while performing "pure, basic research" was negatively associated with policy engagement. For acting as a decision maker, high self-perceived competence, being older, and being male had strong positive associations, while working in a university or college and having had previous bad experiences while engaging in policy were negatively related.

\section{Sociodemographics}

On the basis of the self-reported frequency of engagement, we found that males tended to report, integrate, and act as a decision maker more often than females. This may reflect systematic institutional and cultural barriers and biases against women in science, as a result of hiring processes (Moss-Racusin et al. 2012), traditional gender roles in work versus family life (O'Brien and Hapgood 2012), and journalistic bias against women (von Roten 2011).

Natural scientists and respondents who are conducting "pure, basic research" were less likely to interpret and integrate science, perhaps because they are unaccustomed to or wary of these activities or because they do not perceive the relevance of their work to policy makers (Steel et al. 2004). More involved forms of engagement may be more accepted in other fields of study, or there may be more opportunities for scientists outside of natural science to engage more frequently in policy issues (Jensen et al. 2008).

\section{Self-perceived competence}

Self-perceived competence in engagement was a strong predictor across all types of policy-related activities and was consistently among the top three most influential variables in this study. Competence also remained an important predictor among our subset of professors. Competence may be a significant factor in reporting science, depending on the different levels of experience and training in policy processes among scientists. For other forms of engagement that can expose a scientist to increased scrutiny and criticism, high self-perceived competence may be associated with greater confidence in one's ability to defend a position, thus leading to a greater willingness to engage. The importance of self-perceived 


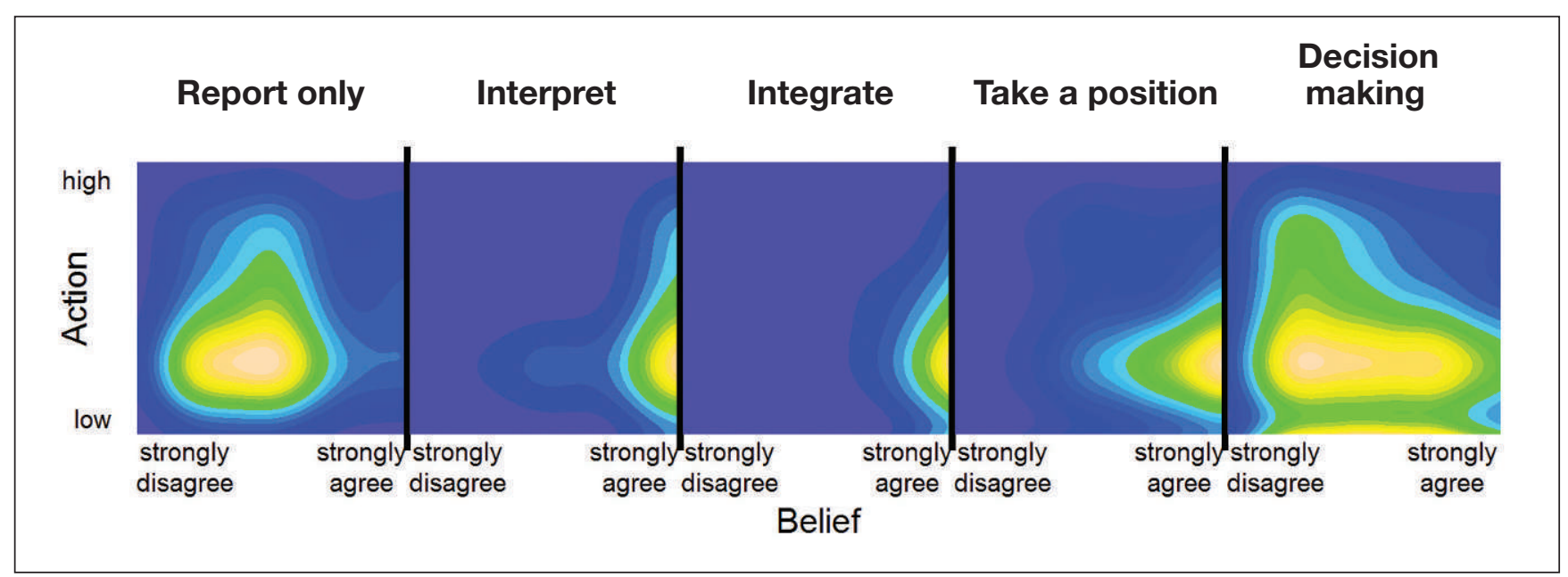

Figure 2. There is a disconnect between beliefs in how scientists should engage in policy making as compared with actual engagement. Shown here is a comparison of respondents' views on how they act (Action) versus how they believe scientists should act (Belief). Within a given type of engagement, note that belief in policy engagement ( $x$ axis; disagree to agree) and level of action in engagement (y axis; low to high) often do not match (ie strongly agreeing that scientists should engage in a certain way often does not coincide with relatively high levels of action). The warmer colors (white, orange, yellow) denote regions of high frequency of response, whereas cooler colors (purple, blue, green) indicate lower frequency.

competence in all forms of policy participation could indicate that programs intended to increase scientists' experience and know-how with policy and communication can lead to higher rates of scientists' involvement, while eliminating biases unrelated to scientific authoritativeness. Conversely, if scientists are more aware of how their work fits into the policy landscape, they may be more likely to get involved.

\section{Public exposure}

For those engagement activities that have higher degrees of public and political exposure (such as taking a position and acting as a decision maker), variables related to public scrutiny were important. Past negative experiences when engaging in policy were a deterrent for both interpreting science and acting as a decision maker. This expanded on a previous finding that scientists with past experience in the science-policy arena are, in general, more likely to get involved again (Poliakoff and Webb 2007).

\section{Tenure status}

Using a subset of the data that included only professors, we observed that tenure consistently had a positive effect on types of participation and was one of the top variables ranked by influence (between the second and sixth most influential variables; WebTable 6). The security and freedom that tenure provides may allow professors the flexibility to get involved in policy making in ways that nontenured professors may not feel comfortable doing (Whitmer et al. 2010). However, the high variability around the coefficient values warrants caution when drawing specific conclusions.
Professors may have diverse reasons for engaging in policy issues besides communicating science, and tenure may offer more freedom to get involved in such activities. Relative to non-tenured professors, tenured professors are 25\% more uncertain (selecting "Maybe") when asked about supporting policies that conflict with their personal values (WebFigure 3) but a high proportion of both tenured and untenured professors ( 50-60\%) were somewhat supportive in backing policies that lack scientific support (indicating "Yes" or "Maybe"; WebFigure 4), which may indicate a willingness to consider values in addition to sound science when endorsing policy. These results highlight the complex influences that may affect scientists' engagement in policy and that warrant future research.

\section{Strong support for engagement, even advocacy}

Our results demonstrate a prevailing belief among those in environmental science and policy that scientists should engage in science interpretation, integration, and even advocacy (Figure 2). This is in strong contrast to the polarized views found in the literature. A previous survey also found less support for the types of engagement we explored (Lach et al. 2003). Scientists in that study felt that fellow scientists should not engage in activities other than interpreting and integrating science (Lach et al. 2003). These differences may be due to our broader sample and the different phrasing and context of the questions in the two surveys. Also, sentiments have possibly changed in the decade between the surveys. In our questionnaire, when asked under what conditions respondents would advocate for science, only $2 \%$ indicated that they would not do so, whereas the greatest proportion $(49 \%)$ suggested that, should research findings agree with 
a policy position, they would be willing to advocate (WebFigure 5).

\section{Frequency of engagement is discordant with belief}

We also find a disconnect between respondents' expressed desire to engage in policy and the degree to which they actually do so (the majority engage in such activities one to three times per year; Figure 2). When prompted with the statement that scientists should only report results without taking further action (ie without interpreting, integrating, advocating, or deciding), participants exhibit a wide variation in responses (from strongly disagree to strongly agree), but involvement in these activities remains relatively low.

In response to a question about how much time participants would want to dedicate to engagement, $62 \%$ indicated that they would like to spend more time than they do now, 36\% said they would dedicate about the same amount of time as they currently do, and only $2 \%$ said they would spend less time than they spend at present. When asked what percentage of their time is dedicated to involvement in policy-related activities, the most common response was $1-10 \%$ of time $(33 \%)$, followed by $11-20 \%$ of time $(18 \%)$, then by $21-30 \%(12 \%)$, with smaller proportions estimating $>30 \%$ (data not shown). Finally, perceived time constraints were not a statistically significant predictor of policy engagement for any of the five types of activities.

This disconnect has many potential explanations. While it has been hypothesized that science institutions may not allow enough time for their personnel to engage as much as they would like to (Whitmer et al. 2010; Bauer and Jensen 2011), our results suggest low levels of policy involvement are not easily explained by time availability. Instead, institutional and cultural norms may more often generate perceived barriers (Whitmer et al. 2010). Many people who engage in policy at "arm's length" (from reporting to integrating science without taking a position) might feel uncomfortable with "advocacy" and its connotations. Notably, the time or seniority required for some forms of engagement (eg interpretation, integration, and especially advocacy) is perhaps lower than for reporting or serving as a decision maker. For example, attending a rally or signing a petition (both forms of policy advocacy) require less time than writing a science brief for a policy maker (integrating), and both require much less time than publishing a peer-reviewed article (reporting). Despite the apparent widespread desire to engage beyond reporting results and despite the relatively low hurdles that time availability and seniority seem to present for policy participation, activity levels for interpretation, integration, and policy advocacy are nevertheless low.

\section{Conclusions}

This study provides a much-needed empirical foundation for strategies to overcome perceived barriers at the science-policy interface. The strong positive association of competence, and the negative association of previous poor experiences, with policy engagement suggest that training programs in science communication and policy may increase scientists' involvement with policy. This may occur by preventing or minimizing negative experiences, increasing competence, and increasing scientists' awareness of how their work is relevant to policy decisions. Training targeted at different modes of participation may also be warranted, given that different types of engagement (ie reporting, interpreting, integrating, taking a position, acting as a decision maker) are constrained by different barriers and enhanced by different incentives (eg natural scientists may require special training in integrating as compared with scientists in other fields, given that natural scientists engage in integrating less frequently). Across all forms of policy engagement, there is a latent desire to engage more frequently. Our empirical results identify possible points of intervention to better align the actions of environmental scientists with their desire to play an important role in the environmental decisionmaking arena.

\section{Acknowledgements}

This project was funded by a Canadian Foundation for Innovation (Leaders Opportunity Fund) grant \#F070010, The Nature Conservancy grant \#08-0687, and a small institutional grant through the College for Interdisciplinary Studies, grant \#F11-02226. GGS, JT, and SCK thank the Natural Science and Engineering Council of Canada, the Social Science and Humanities Research Council of Canada, the University of British Columbia (UBC), and the Pacific Institute for Climate Solutions for financial support. TDS thanks IRES and Green College for hosting his sabbatical at UBC, and M Olajos for supporting work at the science-policy interface. C Millar provided support inputting, coding, and managing the database. $\mathrm{R}$ Naidoo provided statistical guidance.

\section{References}

Bauer MW and Jensen P. 2011. The mobilization of scientists for public engagement. Public Underst Sci 20: 3-11.

Burnham KP and Anderson D. 2002. Model selection and multimodel inference: a practical information-theoretic approach. New York, NY: Springer.

Calcagno V. 2012. glmulti: model selection and multimodel inference made easy. $\mathrm{R}$ package version 1.0.4. http://cran. r-project.org/web/packages/glmulti/index.html. Viewed 10 Oct 2013.

Chan KMA, Higgins PAT, and Porder S. 2005. Protecting science from abuse requires a broader form of outreach. PLoS Biol 3: e218.

Gelman AG. 2008. Scaling regression inputs by dividing by two standard deviations. Stat Med 27: 2865-73.

Gray NJ and Campbell LM. 2009. Science, policy advocacy, and marine protected areas. Conserv Biol 23: 460-68. 
Jensen P, Rouquier JB, Kreimer P, et al. 2008. Scientists who engage with society perform better academically. Sci Public Policy 35: 527-41.

Lach D, List P, Steel B, and Shindler B. 2003. Advocacy and credibility of ecological scientists in resource decisionmaking: a regional study. BioScience 53: 170-78.

Lackey RT. 2007. Science, scientists, and policy advocacy. Conserv Biol 21: 12-17.

Moss-Racusin CA, Dovidio JF, Brescoll VL, et al. 2012. Science faculty's subtle gender biases favor male students. P Natl Acad Sci USA 41: 16474-79.

Nielsen LA. 2001. Science and advocacy are different - and we need to keep them that way. Hum Dimens Wildlife 6: 39-47.

Noss R. 2007. Values are a good thing in conservation biology. Conserv Biol 21: 18-20.

O'Brien KR and Hapgood KP. 2012. The academic jungle: ecosystem modelling reveals why women are driven out of research. Oikos 121: 999-1004.

Pielke RA. 2007. The honest broker: making sense of science in policy and politics. New York, NY: Cambridge University Press.

Poliakoff E and Webb TL. 2007. What factors predict scientists' intentions to participate in public engagement of science activities? Sci Commun 29: 242-63.

Royal Society. 2006. Survey of factors affecting science communication by scientists and engineers. London, UK: Royal Society.

Scott JM, Rachlow JL, Lackey RT, et al. 2007. Policy advocacy in science: prevalence, perspectives, and implications for conservation biologists. Conserv Biol 21: 29-35.

Steel B, List P, Lach D, et al. 2004. The role of scientists in the environmental policy process: a case study from the American West. Environ Sci Policy 7: 1-13.

von Roten FC. 2011. Gender differences in scientists' public outreach and engagement activities. Sci Commun 33: 52-75.

Whitmer A, Ogden L, Lawton J, et al. 2010. The engaged university: providing a platform for research that transforms society. Front Ecol Environ 8: 314-21.

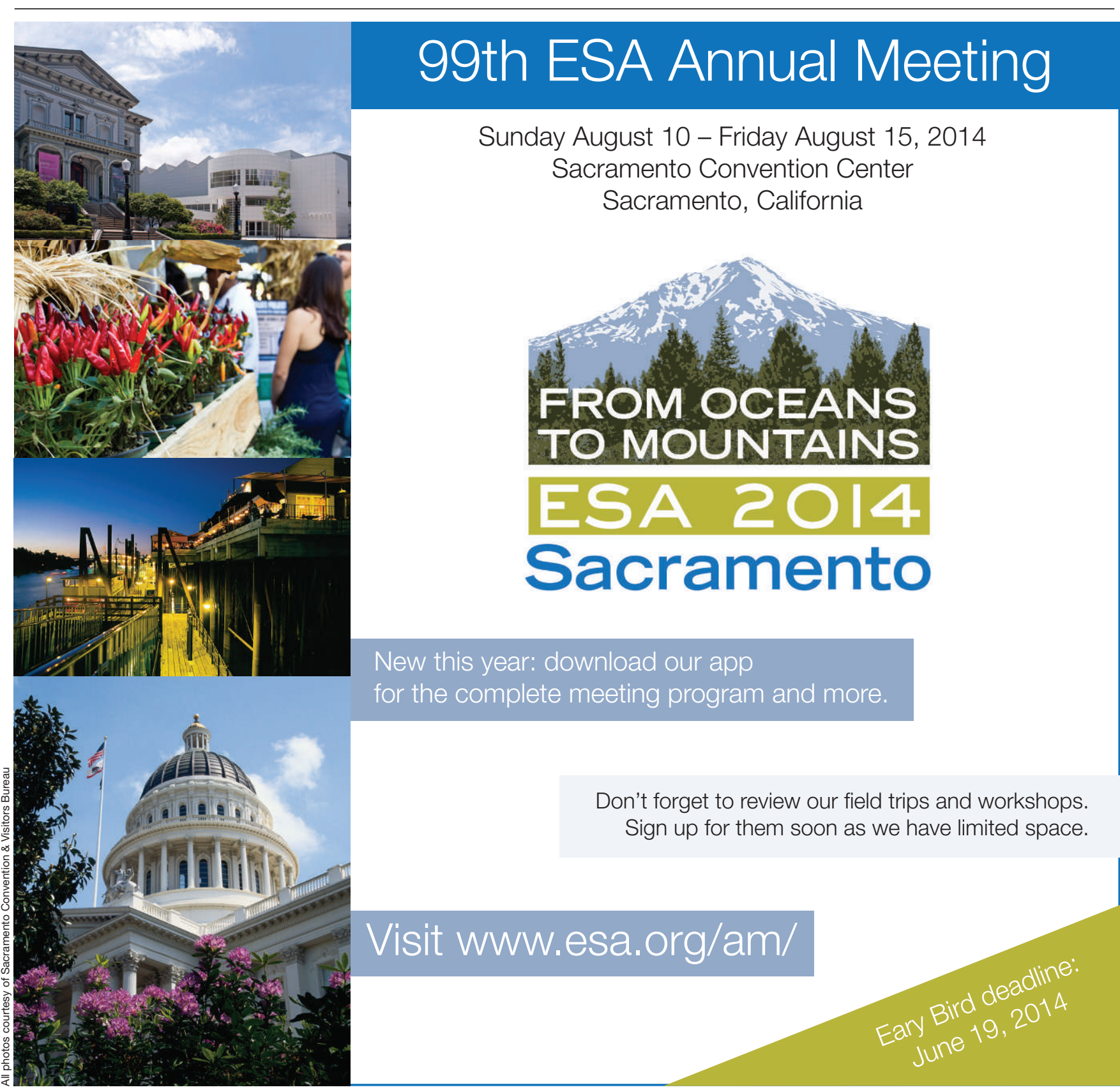

